\title{
Teacher's Perception on Children Having Attention Deficit Hyperactive Disorder (ADHD)
}

\author{
Mahewash Hasan ${ }^{1}$, Nishi Tripathi ${ }^{2, *}$ \\ ${ }^{1}$ Chitamber School of Humanities and Social Sciences, Allahabad-211007 \\ ${ }^{2}$ Sam Higginbottom Institute of Agriculture, Technology and Sciences (SHIATS), \\ (Formerly Allahabad Agricultural Institute), Allahabad-211007 \\ *Corresponding Author:tripathi_nishi@yahoo.com
}

Copyright (C) 2014 Horizon Research Publishing All rights reserved.

\begin{abstract}
The purpose of this study was to investigate teachers' perceptions of children in their classes for which an instrument or a checklist for teachers was developed to help in the identification of ADHD in children. Problem Behavior Rating Scale for teachers was developed and its reliability was measured. Analysis of the data was performed to describe teachers' perceptions of children on the basis of class, age and gender of children with ADHD disorder. It was found that there were age and gender differences in children with ADHD. There was class wise difference also in children with ADHD.
\end{abstract}

Keywords Problem Behavior Rating Scale, Children with ADHD

\section{Introduction}

The present study focuses on attention deficit hyperactivity disorder (ADHD) a common neurobehavioral disorder affecting children and causing significant limitations in functioning across different settings especially school setting. The cardinal symptoms of the disorder include inappropriate levels of inattentiveness, motor over activity and impulsivity. The major concern to conduct a study in this area was to prevent children with ADHD to grow into an impulsive, aggressive and chaotic adult. The disorder is one of the most commonly diagnosed childhood disorders in India (Crawford, S.G. 2007). The average age at which the children were diagnosed was 11.36 years (with a range from 7 to 17 years), while the average age at which the children's symptoms had first been noticed was only 5.55 years (with a range from 4 to 6 years). The delay between symptoms first being noticed and the child being diagnosed with SpLD and ADHD was nearly 6 years on average for the children studied by Karande, et al. Thus, it is not surprising that all of the children in their study demonstrated poor school performance by the time they were assessed in the authors' clinic. Problems in school performance, as opposed to specific symptoms of ADHD, are common complaints and common reasons for referral to child development centers in India (Wilcox, C.E., Washburn, R., Patel, V. 2007) The purpose of the study was to recognize the importance of teachers in identification of children with ADHD and to help in diagnosis since teachers are often the first to notice that a child is having problems at school. Children with ADHD have academic underachievement, poor academic performance, and educational problems. Other classroom behavior may include problems with school work, including problems finishing assignments, organizing work, or staying on task, problems remembering to bring books and materials to class, problems listening to instruction.

Keeping in mind the role of teachers in identifying children with ADHD, a checklist, Problem Behaviour Report Form ' $A$ ' was developed by the authors to help the teachers to tab children with ADHD. Another important purpose was to create awareness among teachers relating to ADHD disorder in children of their classes and to sensitize them with problems that such children face. Child behavior checklists and rating scales have become an essential element in the evaluation and diagnosis of children with behavior problems. The availability of several scales with excellent reliable and valid normative data across a wide age range of children makes their incorporation into the assessment protocol quite convenient and extremely useful. Such information is invaluable in determining the statistical deviance of the children's problem behaviors and the degree to which other problems may be present. Numerous child behavior rating scales exist; (Barkley, 1988a, 1990) Behavior rating scales offer a means of gathering information from informants who may have spent months or years with the child. Apart from interviews, there is no other means of obtaining such a wealth of information with so little investment of time.

\section{Aims and Objectives}

1. To develop Problem Behavior Rating Scale for 
teachers to assist in the identification of children having ADHD.

2. To administer the Problem Behavior Rating Scale for teachers on the teachers of children from classes 2 to 8 to help in the identification of children having ADHD.

3. To standardize the Problem Behavior Rating Scale for teachers for further use in identification of ADH D

\section{Review of Literature in the Area of Interest}

With the help of Teacher Rating Scale Sanchez, Emelyn Y. Velarde (2011) found 7.4\%, prevalence of ADHD ( $\mathrm{N}=229)$ in a school sample of children ages 6-11 years in the city of Panama and confirmed that ADHD is overlooked in some cultures and that a significant number of children with ADHD-related impairments are not receiving optimal intervention. In another study Judith R. and others in the year (2010) indicated that behaviors that discriminate children and adolescents with ADHD from those without were associated with primary symptoms, symptoms of comorbid conditions, and indicators of functional impairment. Teachers rated learning problems as the strongest discriminator of adolescents and as the strongest discriminator of children with ADHD from those without. The findings of the study further supported the need for a multidimensional view of ADHD that adds emphasis to issues of functional impairment in routine, daily activities important to success in school and interpersonally. Teacher Ratings showed significant correlations (Naglieri J.A, et al. 2005) between most of the WISC-III factors and the Cognitive Problems/Inattention scores, but correlations between the WISC-III and Conners' Parent Rating Scale were all non-significant when the aim of the study was examine the relationships between intelligence, ratings of behavior, and continuous performance test scores for a sample of 117 children aged 6-16 years who were referred to a specialty clinic. The study establishes the need of teachers to help in the identification of ADHD. Jennifer A. Mautone, et.al, (2011) inferred that children with ADHD and learning disabilities had significantly lower teacher ratings of academic competence. Results of the present study suggest that Homework Performance Questionnaire (HPQ), Parent and Teacher Versions scores may be used to make valid inferences about the homework performance of children with attention problems. These rating scales may be helpful in progress monitoring and evaluating intervention effectiveness. Patricia Mahar, Grand Forks, ND (2007) found significant differences between the perceptions of teachers who had taught one to six years and those that had taught seven or more years. Teachers who taught seven years or more thought students diagnosed with ADHD had trouble playing, but tried as hard in school as other students. They also felt comfortable referring students who displayed ADHD symptoms as compared to teachers who taught one to six years. It makes sense that teachers with more years of teaching have experienced both success and failures when working with students diagnosed with ADHD and have learned from those experiences. In response to the research question pertaining to gender, participants tended to respond similarly to most survey questions. Male teachers did not believe girls typically are diagnosed with ADHD more than boys. This perception is also supported by NIMH (1998) stating that ADHD occurs three times more often in boys than in girls. Male teachers also noted they did not believe students diagnosed with ADHD have difficulty organizing homework or playing.

\section{Methodology}

Sample: The sample consisted of teachers of classes 2 to 8 teaching in schools of Allahabad city. The sample covered ten $\%$ of the schools in Allahabad city which were randomly selected by the researcher. Government schools as well as private schools were selected for the study. Out of ten schools eight schools had English as their medium of teaching while two were Hindi medium schools. The schools randomly selected were of medium socio-economic status.

Table 1. Depicts the Process of Development of Problem Behavior Rating Scale for Teachers

\begin{tabular}{|c|}
\hline Research work on identification of children having ADHD \\
\hline $\begin{array}{c}\text { Development of items encompassing the common behavioral problems } \\
\text { in children of ages 7-14 with special emphasis on ADHD symptoms } \\
\text { relying on the on the basis of DSMIV criteria and from reviews of } \\
\text { existing literature }\end{array}$ \\
Item Analysis \\
\hline ADHD symptoms \\
Formulation of Dimensions \\
depending on the characteristics of ADHD symptoms \\
\hline $\begin{array}{c}\text { Scoring the checklist or Problem Behavior Rating Scale for Teachers } \\
\text { Administration of the checklist to tap the perception of teachers } \\
\text { regarding ADHD in identification of children having ADHD. }\end{array}$ \\
\hline $\begin{array}{c}\text { Standardization of the checklist for identification of children } \\
\text { having ADHD }\end{array}$ \\
\hline $\begin{array}{c}\text { Entered into the second Phase of the Study for the administration and } \\
\text { identification of children having ADHD }\end{array}$ \\
\hline
\end{tabular}

Source: Computed by the authors

\subsection{Problem Behavior Rating Scale for Teachers}

Rationale: The Problem Behavior Rating Scale for Teachers was developed by the researchers on the basis of already existing review and DSM IV criteria to assist in the identification of children having ADHD. The Problem Behavior Rating Scale for Teachers was developed to ensure the appropriateness of the tool with respect to the suitability and feasibility to the behavioral symptoms of ADHD 
Disorder. Problem Behavior Rating Scale for Teachers was used as a tool to assess the intensity and prevalence of the disorder among children of ages 7 to 14 years. It further assisted in analyzing the causes of the disorder in children.

Description: The final shape of the Problem Behavior Rating Scale for Teachers was given which comprised of 44 items out of which 27 items were those hat assessed ADHD symptoms. It was divided into 3 areas (1) Inattentive (2) Hyperactive/Impulsive and (3) Combined. Inattentive had 13 items and Hyperactive/Impulsivity had 14 items. The other 17 items which inclined more towards conduct disorder and anxiety disorder was not scraped from the list. These items would indicate co morbidity in children having ADHD disorder. These items would also increase the credibility of the Problem Behavior Rating Scale for Teachers since it would separate ADHD with other behavioral problems found in children going to school. Each item had dichotomous response 'yes or no', the teachers were asked to go through each item carefully before responding on each item. If the response was yes then the responses on the Problem Behavior Rating Scale for Teachers were scaled on three points. The authenticity of the identification on the scale increased since only those items were included that were rated as two or three. Children were labeled as having ADHD only when more than $50 \%$ of the items were rated as two or three on three point rating scale. For the labeling of the subtypes the child was supposed to receive as two or three on rating scale on more than 8 items on the dimension of Inattention or Hyperactivity /Impulsivity.

\subsection{Procedure}

As the first phase of the study was designed to develop a checklist which was named as Problem Behavior Rating Scale for Teachers to be filled by the teachers of classes 2 to 8 that would be a reliable source of identifying children having ADHD, the researcher randomly selected 10 schools from Allahabad city of medium socio economic status. There are approximately 50 schools registered under ICSE and CBSE boards and 54 schools of UP boards which are government run schools. The schools were randomly selected in a manner that it covers the whole city of Allahabad. On the basis of the prior permission from the school authority demographic information about the school like its name, date of its establishment, location, level of school, medium of teaching total number of students, total number of staff, total number of classes and number of sections in each class, and socio economic status of the school was gathered from the school office.

The next step was to conduct a seminar for the teachers of classes 2 nd to 8 th on the symptoms and importance of awareness about ADHD. The seminar helped to sensitize teachers about the problems of children with ADHD. The teachers were then asked to prepare a list of students having behavioral problems. For each child identified, the teachers were asked to fill the Problem Behavior Rating Scale for
Teachers. The checklist was also translated in Hindi keeping in view the maximum population speaking Hindi. They were further instructed to clarify their doubts before they move ahead since it was a very sensitive matter and the researcher was cautious to see that teachers made no mistake in responding. The researcher was very vigilant throughout the study so that the ethical issues were kept into considerations. Demographic information about the teacher and the child identified by the teachers was collected from the teachers who participated in the study. The same process was repeated in each school.

\section{Results}

The Data of the present study was analyzed keeping in view with the following research questions:

1. To develop Problem Behavior Rating Scale for Teachers to identify children having ADHD

2. Age wise difference between the two age groups as regards to the symptoms of inattention and hyperactivity.

3. Class wise prevalence of ADHD among children from classes 2-8 with respect to the teacher's perception

4. To examine the possible gender differences in ADHD among the children with respect to the teachers perception

5. To examine the possible age differences in ADHD among the children with respect to the teachers perception

Problem Behavior Rating Scale for Teachers to identify children having ADHD was developed by the researchers and was successfully used for identifying and diagnosing children having ADHD. The steps involved are displayed in table no. 1 .

Table 2. Mean Comparison of Symptoms on the basis of Age

\begin{tabular}{|c|c|c|c|}
\hline \multirow{2}{*}{ Symptoms } & Mean & Mean & \multirow{2}{*}{ t-value } \\
\cline { 2 - 3 } & G1 (7-11) & $\begin{array}{c}\text { Age N=524 } \\
\text { G1 (12-14) }\end{array}$ & \\
\hline Inattention & 12.57 & 13.18 & $44.53 * * *$ \\
\hline Hyperactive & 15.87 & 15.55 & $69.43^{* * *}$ \\
\hline
\end{tabular}

$\mathrm{P}$-value $<.001$

The above table No. 2 shows the Mean Comparison of Variables, t-value and its significance. The findings of the table depicts that cases of inattention were more in the age range of 12-14 than in the other group which shows that children in the age group of 12-14 were more inattentive than in the age group of 7-11. It further shows that the children in the age range 7-11 were more hyperactive than the children in the age range of 12-14. 


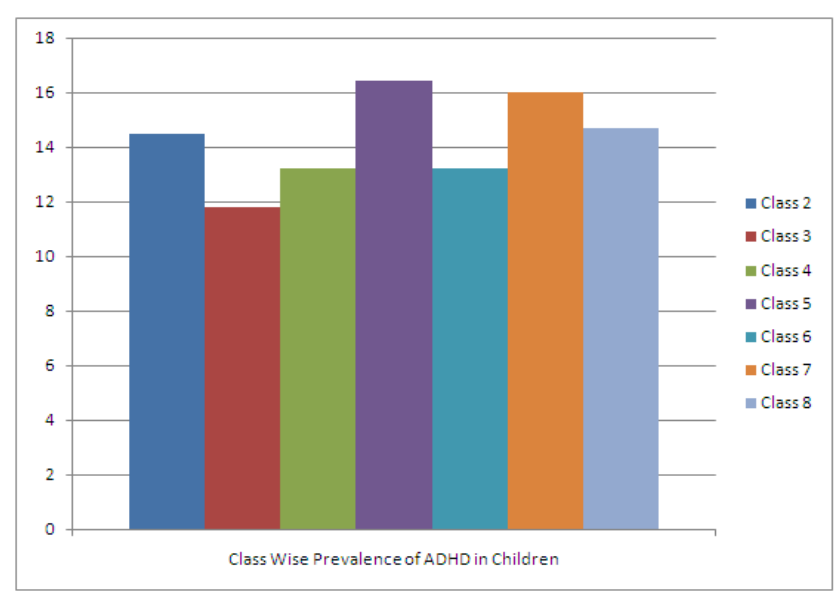

Figure 1. Class Wise Prevalence of ADHD in Children

The above Figure No.1 depicts Class Wise Prevalence of ADHD in children. The finding further indicates that class 5th had maximum (16.4\%) percentage of children with ADHD followed by class 7 th (16\%) of children with ADHD. Next was class 8 th (14.7\%) then class 2 had $14.2 \%$ of children with ADHD and classes 4th and 6thhad $13.2 \%$ of children with ADHD. Least percentage (11.8\%) of children with ADHD belonged to class 3 .

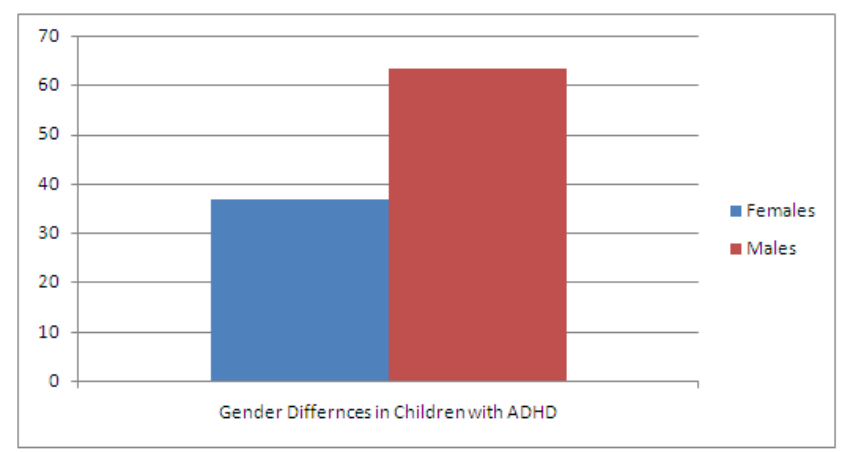

Figure 2. Gender Differences in Children with ADHD

Figure No. 2 shows Gender Differences in Children with ADHD. The finding of the figure 3 shows that ADHD was more prevalent in boys which was $(63.2 \%)$ was more than girls (36.8\%).

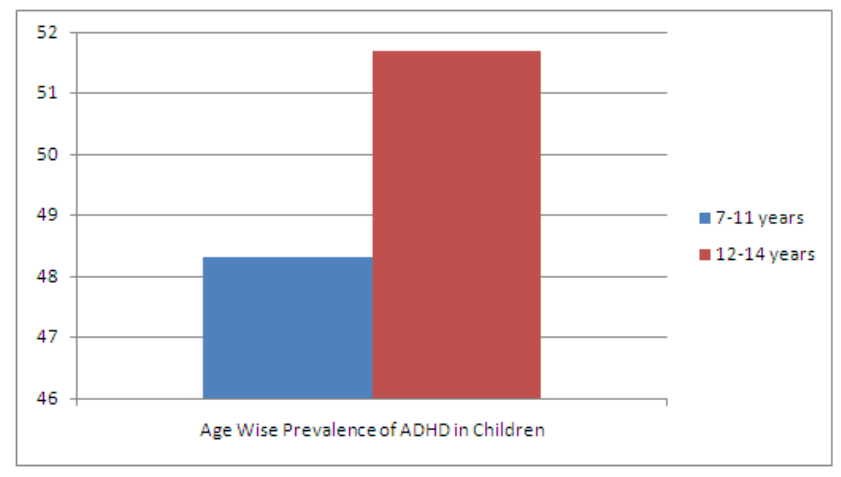

Figure 3. Age Wise Prevalence of ADHD in Children

Figure no. 3 shows age wise Prevalence of ADHD in
Children. Findings of the figure suggest that $51.7 \%$ of children having ADHD belonged to the age group of $7-11$ years and while $48.3 \%$ of children having ADHD belonged to the age group of 12-14 years which means that there were more ADHD children in age group of 7-11years than in age group of 12-14 years.

\section{Discussion}

By applying the Problem Behavior Rating Scale for Teachers to identify children having ADHD, the present study estimates the prevalence ADHD in male and female children. Strengths of this study include the wide age range and large sample size, which provide enough power to examine gender and age differences in great detail. The study was conducted to develop a checklist to help in the identification of children having ADHD. To meet the said target the Problem Behavior Rating Scale for Teachers consisting of 45 items was developed. Table 1 describes how Problem Behavior Rating Scale for Teachers was developed for the purpose. Each step involved in its development is presented in a table form. Significant difference was found between two age groups as regards to ADHD symptoms. Cases of inattention were more in the age range of 12-14 than in the other group which shows that children in the age group of 12-14 were more inattentive than in the age group of 7-11. It further shows that the children in the age range 7-11 were more hyperactive than the children in the age range of 12-14. Cuffe SP, Moore CG, McKeown RE concluded that ADHD symptoms vary by race and ethnicity, gender, and age and are associated with other emotional and behavioral difficulties.

Another question met by the researchers was class wise prevalence of ADHD in children. The result (Figure1) showed that ADHD in children can be detected in children very early because in the present study $14.5 \%$ children belonged to the 2 th standard. It could be foreseen that if the school authorities and parents are sensitive towards the behavioral problems of children then the early detection can help to control the problems in children. Medication or psychotherapeutic intervention could be started at the earliest because approximately 5 to 10 percent of all school-age children worldwide have ADHD, and the disorder interferes with schoolwork and the interaction with classmates and peers. Longitudinal studies show that some children who are diagnosed with ADHD continue to exhibit the symptoms into adulthood. Maximum number of children having ADHD was found in class 5 th with $16.4 \%$. The behaviors associated with ADHD may be first observed or most troublesome in a classroom setting. Snider, Frankenberger, and Aspensen (2000) found that teachers were involved in making the initial referral for a special education evaluation nearly $60 \%$ of the time. This suggests that teachers play an important role in the initial screening for ADHD. In light of this, it is critical that teachers are knowledgeable and objective if they are to play a key role in the diagnosis and education of students 
with ADHD.

Yet another question in the study was to examine the possible gender differences in ADHD. In the present study it was found that there were $63.2 \%$ boys having ADHD and $36.8 \%$ were girls having ADHD disorder (figure2). Previously study by José J.Bauermeister, Patrick E. Shrout \& others in (2007) have found that ADHD was 2.3 times more common in boys than girls. According to Julia J. Rucklidge (2010) that attention-deficit hyperactivity disorder (ADHD) is recognized to exist in males and females although the literature supports a higher prevalence in males. In yet another study conducted by Jonathan Gershon (2002) examined gender differences in ADHD through a meta-analysis and the results indicated that in comparison to ADHD boys, ADHD girls had lower ratings on hyperactivity, inattention, impulsivity, and externalizing problems. The sex distribution of all the children attending clinic in a study by M. S. Bhatia S. Choudhary Ajeet Sidana (1998) was 58\% boys and $42 \%$ girls (1.4:I) whereas the boy: girl ratio of $3: 1$ among children with ADHD was significantly different from that in the sex distribution of children in the outpatient clinic.

The total sample of children was divided into two groups: 7-11years and 12-14 years. Children in the 12-14 years group had more ADHD cases than the other group(figure3). It could be concluded from analysis that the disorder was evenly spread through the ages from 7 years to 14 years. Of the diagnosed children, $19.6 \%$ were less than 3 years, $35.3 \%$ of children were between 3 and 5 years of age, $41 \%$ were between 5 and 9 years of age and $12 \%$ were above 9 years of age. The mean age for boys was 5.8 years $(\mathrm{SD} \pm 2.9)$. The mean age for girls was 4.9 years $(\mathrm{SD} \pm 1.9)$ Venkatesh et al. (2012).

\section{Conclusion}

Our study provides a broad view of ADHD in children and the utility of teachers rating scale in the identification and diagnosis of ADHD in children. The study further states that Teachers' perception plays an important role in the identification of children having ADHD. The result shows that ADHD is more common in males than in females. The result further shows that there is not much class wise difference in the prevalence of ADHD. Problem Behavior Rating Scale for Teachers helps in the early detection of ADHD in children. Awareness and sensitization program are necessary for teachers and parents to help children having ADHD. This study has attempted to explore the validity of teachers rating scale as a fair diagnostic instrument on India teachers. It was considered vital to explore the problems associated with ADHD in children to successfully address the educational needs within

\section{Limitations}

Study was conducted only in Allahabad. And hence it cannot be highly generalized.

\section{Implications}

Our study shows the utility of teacher's response on Problem Behaviour Rating Scale Form 'A' children with ADHD and suggests that awareness among teachers and timely detection may be a valuable tool to manage these problems in ADHD children. Inattention and hyperactivity symptoms are common classroom complaints and therefore the result of this study can be important for the welfare of children with ADHD. Age and gender may be considered as major determinants of ADHD in children. The present study opens new opportunities for researchers to work on ADHD children struggling with daily activities.

\section{REFERENCES}

[1] Bhatia, M.S., Choudhary S. \&SidanaAjeet (1998). www.scribd.com/doc/.../About-Case-Studies-of-ADHD-in-C hildren (2011)

[2] Barkley, R. A. (1988). Child Behavior Rating Scales and Checklists. In M. Rutter, H. Tuma, \& I. Lann (Eds.), Assessment and diagnosis in child psychopathology. (pp. 113-155). New York: Guilford Press

[3] Crawford SG.(2007) Specific Learning Disabilities and Attention-Deficit Hyperactivity Disorder: Under-recognized in India. Indian J Med Sci 2007;61:637-8

[4] Cuffe SP, Moore CG, McKeown RE. (2005) Journal of Attention Disorders. 2005 Nov;9(2):392-401.

[5] Guyer, B. P. (2002). ADHD achieving success in school and in life. Needham Heights, MA: Allyn \& Bacon

[6] Jonathan Gershon(2002). Journal of Attention Disorders vol. 5 no. 3 143-154

[7] José J. Bauermeister, Patrick E. Shrout\& others (2007). Journal of Abnormal Child Psychology Volume 21, Number 5 (1993), 519-533, DOI: 10.1007/BF00916317

[8] Julia J. Rucklidge (2010). Psychiatric Clinics of North America Volume 33, Issue 2 , Pages 357-373, June 2010

[9] Karande S, Satam N, Kulkarni M, Sholapurwala R, Chitre A, Shah N.(2007) Clinical and Psychoeducational profile of Children with Specific Learning Disability and co-occurring Attention-Deficit Hyperactivity Disorder. Indian J Med Sci 2007;61:639-47.

[10] M.S. Bhatia S. ChoudharyAjeetSidana (1999) Indian Pediatrics;36:583-587

[11] Patricia Mahar, Grand Forks, ND (2007) National Forum Of Applied Education Research Journal Volume 20, Number 3,2007

[12] Snider, V. E., Frankenberger, W., \&Aspenson, M. (2000).The Relationship between Learning Disabilities and Attention Deficit Hyperactivity Disorder: A National Survey. 
Developmental Disabilities Bulletin, 28, 18-38

[13] Neuwirth, S. (1996). Attention deficit hyperactivity disorder (NIH Publication No. 96-3572). (Available from NIMH Public Inquiries, 6001 Executive Boulevard, Room 8184 MCS 9663, Bethesda, MD 20892-9663).

[14] Venkatesh C, Ravikumar T, Andal A, Virudhagirinathan B.
S.,(2012). Attention deficit hyperactivity Disorder in Children: Clinical Profile and Comorbidity. Indian Journal of Psychological Med 2012;34:34-8

[15] Wilcox CE., Washburn R, Patel V. (2007). Seeking help for attention deficit hyperactivity disorder in developing countries: A study of parental explanatory models in Goa, India. Soc Sci Med 2007;64:1600-10. 